\title{
Sum Rules Giving Tests of Local Current Commutation Relations in High-Energy Neutrino Reactions
}

\author{
STEPHEN L. ADLER* \\ CERN, Geneva, Switzerland and Lyman Laboratory, Harvard University, Cambridge, Massachusetts
}

(Received 6 October 1965)

\begin{abstract}
We show that the local commutation relations of the vector and the axial-vector current octets can be studied in nonforward lepton-neutrino reactions. We do this by using the commutation relations to derive sum rules, for fixed $q^{2}\left(q^{2}=\right.$ invariant lepton momentum transfer squared), involving the elastic and the inelastic form factors measured in high-energy neutrino reactions.
\end{abstract}

\section{INTRODUCTION}

$I^{T}$ $\mathrm{T}$ has recently been proposed by Gell-Mann ${ }^{1}$ that the fourth components of the vector and axial-vector current octets satisfy the local equal-time commutation relations

$$
\begin{aligned}
& {\left.\left[\mathscr{F}_{a 4}(x), F_{b 4}(y)\right]\right|_{x_{0}=y_{0}}=-f_{a b c} \mathfrak{F}_{c 4}(x) \delta(\mathbf{x}-\mathbf{y}),} \\
& {\left.\left[\mathfrak{F}_{a 4}(x), F_{b 4}{ }^{5}(y)\right]\right|_{x_{0}=y_{0}}=-f_{a b c} \mathfrak{F}_{c 4}{ }^{5}(x) \delta(\mathbf{x}-\mathbf{y}),} \\
& {\left.\left[\mathfrak{F}_{a 4}{ }^{5}(x), F_{b 4}{ }^{5}(y)\right]\right|_{x_{0}=y_{0}}=-f_{a b c} \mathfrak{F}_{c 4}(x) \delta(\mathbf{x}-\mathbf{y}) .}
\end{aligned}
$$

Here $\mathfrak{F}_{a \lambda}$ and $\mathfrak{F}_{a \lambda}{ }^{5}$ are, respectively, the octet vector, and axial-vector currents, and $a, b, c$ are unitary spin indices running from 1 to 8. According to Eq. (1), the octet vector and axial-vector charges

$$
\begin{aligned}
F_{a}(t) & =-i \int d^{3} x \mathcal{F}_{a 4}(\mathbf{x}, t), \\
F_{a}^{5}(t) & =-i \int d^{3} x F_{a 4}{ }^{5}(\mathbf{x}, t),
\end{aligned}
$$

satisfy the equal-time commutation relations

$$
\begin{aligned}
{\left[F_{a}(t), F_{b}(t)\right] } & =i f_{a b c} F_{c}(t), \\
{\left[F_{a}(t), F_{b}^{5}(t)\right] } & =i f_{a b c} F_{c}^{5}(t), \\
{\left[F_{a}^{5}(t), F_{b}^{5}(t)\right] } & =i f_{a b c} F_{c}(t) .
\end{aligned}
$$

The commutation relations of Eq. (1) are considerably more restrictive than those of Eq. (3), since even if derivatives of the delta function were present on the right-hand side of Eq. (1), Eq. (3) would still be valid. In an earlier paper $^{2}$ [hereafter referred to as (I)] we showed that the commutation relations of Eq. (3) can be tested in high-energy inelastic neutrino reactions, in which the lepton (which is regarded as massless) emerges moving parallel to the direction of the incident neutrino. In other words, Eq. (3) may be tested in $q^{2}=0$ neutrino reactions, where $q^{2}$ is the invariant momentum transfer between the neutrino and the outgoing lepton. In this paper we generalize the results of (I), by showing that the local commutation relations of Eq. (1) can be tested in $q^{2}>0$ (nonforward lepton) neutrino reactions. We do this by deriving from Eq. (1) a sum rule, valid for each fixed $q^{2}$, involving quantities measurable in high-energy neutrino reactions.

\footnotetext{
* Junior Fellow, Society of Fellows.

1 M. Gell-Mann, Physics 1, 63 (1964)

2 S. L. Adler, Phys. Rev. 140, B736 (1965).
}

In addition to Eq. (1) for the fourth components of the current octets, let us postulate that the space components of the octets satisfy the local equal-time commutation relations

$$
\begin{aligned}
& {\left.\left[\mathcal{F}_{a n}(x), F_{b m}(y)\right]\right|_{x_{0}=y_{0}}} \\
& =\delta_{n m} f_{a b c} \mathrm{U}_{c 4}{ }^{1}(x) \delta(\mathbf{x}-\mathbf{y})+S_{a b}{ }^{1}, \\
& \left\{\left.\left[\mathscr{F}_{a n}(x), \mathscr{F}_{b m}{ }^{5}(y)\right]\right|_{x_{0}=y_{0}}+\left.\left[\mathscr{F}_{a n}{ }^{5}(x), F_{b m}(y)\right]\right|_{x_{0}=y_{0}}\right\} \\
& =-2 \delta_{n m} f_{a b c} \mathfrak{Q}_{c 4}(x) \delta(\mathbf{x}-\mathbf{y})+S_{a b}{ }^{2}, \\
& {\left.\left[\mathcal{F}_{a n}{ }^{5}(x), \mathscr{F}_{b m}{ }^{5}(y)\right]\right|_{x_{0}=y_{0}}} \\
& =\delta_{n m} f_{a b c} \mathcal{V}_{c 4}{ }^{2}(x) \delta(\mathbf{x}-\mathbf{y})+S_{a b^{3}} .
\end{aligned}
$$

Here $\mathcal{V}_{c 4}{ }^{1}$ and $\mathcal{V}_{c 4}{ }^{2}$ are the fourth components of vectorcurrent octets, and $Q_{c 4}$ is similarly the fourth component of an axial-vector octet. The quantities $S_{a b}{ }^{1,2,3}$ are symmetric in the unitary spin indices $a$ and $b$. If the simple quark-model commutation relations proposed by Dashen and Gell-Mann ${ }^{3}$ and by Lee ${ }^{4}$ are valid, we have

$$
\mathcal{V}_{c 4}{ }^{1}=\mathcal{V}_{c 4}{ }^{2}=F_{c 4}, \quad Q_{c 4}=\mathscr{F}_{c 4}{ }^{5} .
$$

However, Eq. (5) is not valid in theories in which meson fields are explicitly included in the currents, whereas, in many of these field theories, Eq. (4) still holds. We will derive sum rules which provide tests of Eq. (4) in $q^{2}>0$ neutrino reactions.

Each of the sum rules discussed in this paper requires for its derivation, in addition to a local equal-time commutation relation, the assumption that a certain scattering amplitude obeys an unsubtracted dispersion relation in the energy variable, for fixed $q^{2}$. No attempt will be made in this paper to justify the assumption of unsubtracted dispersion relations. Thus, the statement made in this paper is that if the assumption of unsubtracted dispersion relations is valid, the sum rules derived provide a direct experimental test of local equal-time commutation relations.

In Sec. 2 we state in detail the results of the paper. The next two sections comprise the derivation. In Sec. 3 we analyze the kinematics of high-energy neutrino reactions. In Sec. 4 we derive, from local commutation relations, sum rules which involve the quantities defined in the kinematic analysis of Sec. 3. In an Appendix we give lepton-mass corrections to the results stated in Sec. 2.

${ }^{3}$ R. F. Dashen and M. Gell-Mann, Phys. Letters 17, 142 (1965).

${ }^{4}$ B. W. Lee, Phys. Rev. Letters 14, 676 (1965). 


\section{RESULTS}

We consider the high-energy neutrino reaction

$$
\nu+N \rightarrow l+\beta,
$$

where $\nu$ is a neutrino, $N$ is a nucleon (neutron or proton), $l$ is an electron or muon, and $\beta$ is a system of strongly interacting particles. Throughout the text of this paper, we will neglect the final lepton mass, i.e., we take

$$
m_{l} \approx 0 \text {. }
$$

The results stated below are only slightly modified when all lepton-mass terms are included. (See the Appendix.) We define all noninvariant quantities referring to the reaction of Eq. (6) in the laboratory frame, in which the nucleon $N$ is at rest:

$E_{\nu}=$ neutrino energy,

$E_{l}=$ lepton energy,

$\phi=$ lepton-neutrino scattering angle,

$\Omega_{l}=$ lepton solid angle,

$k_{\nu}=$ neutrino four-momentum,

$k_{l}=$ lepton four-momentum,

$q=k_{\nu}-k_{l}=$ lepton four-momentum transfer.
We denote by $W$ the invariant mass of the system $\beta$, by $M_{N}$ the nucleon mass, and by $q^{2}$ the invariant momentum transfer between the leptons:

$$
\begin{aligned}
q^{2} & =\left(k_{\nu}-k_{l}\right)^{2}=4 E_{\nu} E_{l} \sin ^{2}(\phi / 2), \\
W & =\left[2 M_{N}\left(E_{\nu}-E_{l}\right)+M_{N}{ }^{2}-q^{2}\right]^{1 / 2} .
\end{aligned}
$$

We assume that the semileptonic weak interactions are described by the current-current effective Lagrangian density

$$
\begin{aligned}
& \mathcal{L}(x)=(G / \sqrt{2}) j_{\lambda}(x) J_{\lambda}(x)+\text { adjoint }, \\
& G=1.023 \times 10^{-5} / M_{N}{ }^{2}, \\
& j_{\lambda}(x)=\bar{\psi}_{l}(x) \gamma_{\lambda}\left(1+\gamma_{5}\right) \psi_{\nu}(x) \text {, } \\
& J_{\lambda}(x)=\left(\cos \theta_{C}\right)\left[\mathscr{F}_{1 \lambda}(x)+i \Im_{2 \lambda}(x)+\mathscr{F}_{1 \lambda}{ }^{5}(x)+i \mathfrak{F}_{2 \lambda}{ }^{5}\left(x_{1}\right)\right] \\
& +\left(\sin \theta_{C}\right)\left[\Im_{4 \lambda}(x)+i \Im_{5 \lambda}(x)+\Im_{4 \lambda}^{5}(x)+i \Im_{5 \lambda}{ }^{5}(x)\right] \text {, } \\
& \theta_{C}=\text { Cabibbo angle. }
\end{aligned}
$$

We define the form factors $F_{1}^{V}\left(q^{2}\right), F_{2}^{V}\left(q^{2}\right), g_{V}\left(q^{2}\right)$, $g_{A}\left(q^{2}\right)$, and $h_{A}\left(q^{2}\right)$, which describe elastic neutrino reactions, as follows:

$$
\begin{aligned}
\left\langle N\left(p_{2}\right)\left|\Im_{1 \lambda}(0)+i F_{2 \lambda}(0)\right| N\left(p_{1}\right)\right\rangle & =\left(\left(M_{N} / p_{20}\right)\left(M_{N} / p_{10}\right)\right)^{1 / 2} i \bar{u}_{N}\left(p_{2}\right) \tau^{+}\left[F_{1}^{V}\left(q^{2}\right) \gamma_{\lambda}-F_{2} V\left(q^{2}\right) \sigma_{\lambda_{\eta}} q_{\eta}\right] u_{N}\left(p_{1}\right) \\
& =\left(\left(M_{N} / p_{20}\right)\left(M_{N} / p_{10}\right)\right)^{1 / 2} i \bar{u}_{N}\left(p_{2}\right) \tau^{+}\left[g_{V}\left(q^{2}\right) \gamma_{\lambda}+i F_{2} V\left(q^{2}\right)\left(p_{1}+p_{2}\right) \lambda\right] u_{N}\left(p_{1}\right), \\
& =p_{2}-p_{1}, \quad g_{V}\left(q^{2}\right)=F_{1}{ }^{V}\left(q^{2}\right)+2 M_{N} F_{2} V\left(q^{2}\right), \\
\left\langle N\left(p_{2}\right)\left|F_{1 \lambda}{ }^{5}(0)+i \Im_{2 \lambda}{ }^{5}(0)\right| N\left(p_{1}\right)\right\rangle & \left.=\left(\left(M_{N} / p_{20}\right)\left(M_{N} / p_{10}\right)\right)\right)^{1 / 2} i \bar{u}_{N}\left(p_{2}\right) \tau^{+}\left[g_{A}\left(q^{2}\right) \gamma_{\lambda}-i h_{A}\left(q^{2}\right) q_{\lambda}\right] \gamma_{5} u_{N}\left(p_{1}\right) .
\end{aligned}
$$

Here $\tau^{+}$denotes $\frac{1}{2}\left(\tau^{1}+i \tau^{2}\right)$, with $\frac{1}{2} \tau^{c}(c=1,2,3)$ the nucleon isotopic spin matrices.

Finally, we define the diagonal one-nucleon matrix elements of the operators $\mathcal{V}_{c 4}{ }^{1,2}$ appearing in Eq. (4) as follows

$$
\begin{aligned}
& \left\langle N(p)\left|\mho_{c 4}{ }^{1,2}(0)\right| N(p)\right\rangle=i C_{I^{1,2}}\left\langle\frac{1}{2} \tau^{c}\right\rangle, \quad c=1,2,3 ; \\
& \left\langle N(p)\left|\mho_{84}{ }^{1,2}(0)\right| N(p)\right\rangle=i C_{Y^{1,2}} .
\end{aligned}
$$

If the quark-model commutation relations hold, so that Eq. (5) is valid, then

$$
C_{I^{1,2}}=1, \quad C_{Y}{ }^{1,2}=\frac{1}{2} \sqrt{3} \text {. }
$$

If the quark-model commutation relations are not valid, the values of $C_{I^{1,2}}$ and $C_{Y^{1,2}}$ are not at present known.

We may now state the results of this paper, as follows.

\section{Strangeness-Conserving Case}

The kinematic analysis of Sec. 3 shows that we may write the reaction differential cross section in the form

$$
\begin{aligned}
d^{2} \sigma\left(\left(\begin{array}{l}
\nu \\
\bar{\nu}
\end{array}\right)+p \rightarrow\left(\begin{array}{l}
l \\
\bar{l}
\end{array}\right)+\beta(S=0)\right) / d \Omega_{l} d E_{l}=\frac{G^{2} \cos ^{2} \theta_{C}}{(2 \pi)^{2}} \frac{E_{l}}{E_{\nu}} \\
\times\left[q^{2} \alpha^{( \pm)}\left(q^{2}, W\right)+2 E_{\nu} E_{l} \cos ^{2}\left(\frac{1}{2} \phi\right) \beta^{( \pm)}\left(q^{2}, W\right) \mp\left(E_{\nu}+E_{l}\right) q^{2} \gamma^{( \pm)}\left(q^{2}, W\right)\right] .
\end{aligned}
$$

By measuring $d^{2} \sigma / d \Omega_{l} d E_{l}$ for various values of the neutrino energy $E_{\nu}$, the lepton energy $E_{l}$, and the leptonneutrino angle $\phi$, we can determine the form factors $\alpha^{( \pm)}, \beta^{( \pm)}$, and $\gamma^{( \pm)}$for all $q^{2}>0$ and for all $W$ above threshold.

In Sec. 4 we prove that:

(i) the local commutation relations of Eq. (1a) and Eq. (1c) imply

$$
2=g_{A}\left(q^{2}\right)^{2}+F_{1}^{V}\left(q^{2}\right)^{2}+q^{2} F_{2}^{V}\left(q^{2}\right)^{2}+\int_{M_{N}+M_{\pi}}^{\infty} \frac{W}{M_{N}} d W\left[\beta^{(-)}\left(q^{2}, W\right)-\beta^{(+)}\left(q^{2}, W\right)\right] ;
$$


(ii) the local commutation relations of Eq. (4a) and Eq. (4c) imply

$$
C_{I}{ }^{1}+C_{I}^{2}=\left(1+q^{2} / 4 M_{N}^{2}\right) g_{A}\left(q^{2}\right)^{2}+\left(q^{2} / 4 M_{N}^{2}\right) g_{V}\left(q^{2}\right)^{2}+\int_{M_{N}+M_{\pi}}^{\infty} \frac{W}{M_{N}} d W\left[\alpha^{(-)}\left(q^{2}, W\right)-\alpha^{(+)}\left(q^{2}, W\right)\right] ;
$$

(iii) the local commutation relation of Eq. (4b) implies

$$
\frac{g_{V}\left(q^{2}\right) g_{A}\left(q^{2}\right)}{M_{N}}=\int_{M_{N}+M_{\pi}}^{\infty} \frac{W}{M_{N}} d W\left[\gamma^{(-)}\left(q^{2}, W\right)-\gamma^{(+)}\left(q^{2}, W\right)\right] .
$$

We write

\section{Strangeness-Changing Case}

$d^{2} \sigma\left(\left(_{\bar{\nu}}^{\nu}\right)+(p, n) \rightarrow\left(\begin{array}{l}l \\ \bar{l}\end{array}\right)+\beta\left(\begin{array}{c}S=1 \\ S=-1\end{array}\right)\right) / d \Omega_{l} d E_{l}=\frac{G^{2} \sin ^{2} \theta_{C}}{(2 \pi)^{2}} \frac{E_{l}}{E_{\nu}}$

Then,

$$
\times\left[q^{2} \alpha_{(p, n)}{ }^{( \pm)}\left(q^{2}, W\right)+2 E_{\nu} E_{l} \cos ^{2}\left(\frac{1}{2} \phi\right) \beta_{(p, n)}{ }^{( \pm)}\left(q^{2}, W\right) \mp\left(E_{\nu}+E_{l}\right) q^{2} \gamma_{(p, n)}^{( \pm)}\left(q^{2}, W\right)\right] .
$$

(i) the local commutation relations of Eq. (1a) and Eq. (1c) imply

$$
(4,2)=\int \frac{W}{M_{N}} d W\left[\beta_{(p, n)}^{(-)}\left(q^{2}, W\right)-\beta_{(p, n)}{ }^{(+)}\left(q^{2}, W\right)\right] ;
$$

(ii) the local commutation relations of Eq. (4a) and Eq. (4c) imply

$$
\left[\sqrt{3}\left(C_{Y}{ }^{1}+C_{Y}{ }^{2}\right)+\frac{1}{2}\left(C_{I}{ }^{1}+C_{I}{ }^{2}\right), \sqrt{3}\left(C_{Y^{1}}+C_{Y^{2}}\right)-\frac{1}{2}\left(C_{I}{ }^{1}+C_{I}{ }^{2}\right)\right]=\int \frac{W}{M_{N}} d W\left[\alpha_{(p, n)}{ }^{(-)}\left(q^{2}, W\right)-\alpha_{(p, n)}{ }^{(+)}\left(q^{2}, W\right)\right]
$$

(iii) the local commutation relation of Eq. (4b) implies

$$
(0,0)=\int \frac{W}{M_{N}} d W\left[\gamma_{(p, n)}^{(-)}\left(q^{2}, W\right)-\gamma_{(p, n)}^{(+)}\left(q^{2}, W\right)\right] .
$$

The integrals of Eqs. (18)-(20) have discrete contributions at $W=M_{\Lambda}$ and/or $M_{\Sigma}$ and a continuum extending from $W=M_{\Lambda}+M_{\pi}$ or from $W=M_{\Sigma}+M_{\pi}$ to $W=\infty$. We have not explicitly separated off the discrete contributions to the integrals, as was done in Eqs. (14)-(16) for the strangeness-conserving case. It would, of course, be straightforward to do this.

6. The sum rules of Eqs. (14)-(16) and (18)-(20) hold for each fixed $q^{2}$, provided, as was stated in Sec. 1, that the assumption of an unsubtracted dispersion relation needed to derive each sum rule is valid. When $q^{2}=0$, Eqs. (41) and (43) of the next section show that

$$
\beta(0, W)=\left(4 M_{N}^{2} /\left(W^{2}-M_{N}^{2}\right)^{2}\right) \sum_{\beta, \mathrm{INT}} \sum_{s} \delta\left(k_{\beta 0}+E_{l}-E_{\nu}-M_{N}\right)\left\{\left|\left\langle\beta\left|\partial_{\lambda} J_{\lambda}{ }^{V}\right| N\right\rangle\right|^{2}+\left|\left\langle\beta\left|\partial_{\lambda} J_{\lambda}{ }^{A}\right| N\right\rangle\right|^{2}\right\},
$$

where $J_{\lambda}{ }^{V}$ and $J_{\lambda}^{A}$ are the vector and axial-vector weak currents appropriate to the $\Delta S=0$ or $|\Delta S|=1$ cases (e.g., $J_{\lambda} V=F_{1 \lambda}+i F_{2 \lambda}$ or $\mathscr{F}_{4 \lambda}+i F_{5 \lambda}$ ). Thus, at $q^{2}=0$ Eqs. (14) and (18) are just the forward lepton sum rules derived in (I).

The sum rule on $\beta$ has an interesting consequence for the behavior of neutrino cross sections in the limit of very large neutrino energy $E_{\nu}$. With the aid of Eq. (8), let us write Eqs. (13) and (14) in the form

$$
\begin{gathered}
d^{2} \sigma\left(\left(\begin{array}{l}
\nu \\
\bar{\nu}
\end{array}\right)+p \rightarrow\left(\begin{array}{l}
l \\
l
\end{array}\right)+\beta(S=0)\right) / d\left(q^{2}\right) d q_{0}=\frac{G^{2} \cos ^{2} \theta_{C}}{4 \pi E_{\nu}{ }^{2}}\left[q^{2} \alpha^{( \pm)}+\left(2 E_{\nu}{ }^{2}-2 E_{\nu} q_{0}-\frac{1}{2} q^{2}\right) \beta^{( \pm)} \mp\left(2 E_{\nu}-q_{0}\right) q^{2} \gamma^{( \pm)}\right], \\
2=\int_{\left(q^{2} / 2 M_{N}\right)-}^{\infty} d q_{0}\left(\beta^{(-)}-\beta^{(+)}\right) .
\end{gathered}
$$

The differential cross section $d \sigma / d\left(q^{2}\right)$ is given by

$$
\frac{d \sigma}{d q^{2}}=\int_{\left(q^{2} / 2 M_{N}\right)-}^{E_{\nu}\left(1-q^{2} / 4 E_{\nu}{ }^{2}\right)} d q_{0} \frac{d^{2} \sigma}{d\left(q^{2}\right) d q_{0}} .
$$


The upper limit of integration is fixed by the requirement that $\sin ^{2}(\phi / 2)$ lie between 0 and 1 . Using Eqs. (22)-(24), it is straightforward to prove the following theorem:

Theorem. Suppose that the integrals

are convergent. Then

$$
\int^{\infty} \frac{d q_{0}}{q_{0}^{2}}\left(\alpha^{(-)}-\alpha^{(+)}\right), \quad \int^{\infty} \frac{d q_{0}}{q_{0}}\left(\gamma^{(-)}+\gamma^{(+)}\right), \quad \int^{\infty} d q_{0}\left(\beta^{(-)}-\beta^{(+)}\right)
$$

$$
\begin{aligned}
\lim _{E_{\nu} \rightarrow \infty}\left\{d \sigma(\hat{\nu}+p \rightarrow i+\beta(S=0)) / d\left(q^{2}\right)-d \sigma(\nu+p \rightarrow l+\beta(S=0)) / d\left(q^{2}\right)\right\} & \\
& =\frac{G^{2} \cos ^{2} \theta_{C}}{2 \pi} \int_{\left(q^{2} / 2 M_{N}\right)-}^{\infty} d q_{0}\left[\beta^{(-)}-\beta^{(+)}\right]=\frac{G^{2} \cos ^{2} \theta_{C}}{\pi} .
\end{aligned}
$$

Similar results hold in the strangeness-changing case. Adding the cross sections for the $\Delta S=0$ and the $|\Delta S|=1$ cases to obtain the total cross section, we find

$$
\begin{aligned}
& \lim _{E_{\nu \rightarrow \infty}}\left[d \sigma_{T}(\bar{\nu}+p) / d\left(q^{2}\right)-d \sigma_{T}(\nu+p) / d\left(q^{2}\right)\right]=\left(G^{2} / \pi\right)\left(\cos ^{2} \theta_{C}+2 \sin ^{2} \theta_{C}\right), \\
& \lim _{E_{\nu} \rightarrow \infty}\left[d \sigma_{T}(\bar{\nu}+n) / d\left(q^{2}\right)-d \sigma_{T}(\nu+n) / d\left(q^{2}\right)\right]=\left(G^{2} / \pi\right)\left(-\cos ^{2} \theta_{C}+\sin ^{2} \theta_{C}\right) .
\end{aligned}
$$

Equation (27) is the somewhat surprising statement that, in the limit of large neutrino energy, $d \sigma_{T}(\bar{\nu}+N) / d\left(q^{2}\right)$ $-d \sigma_{T}(\nu+N) / d\left(q^{2}\right)$ becomes independent of $q^{2}$. This result is unchanged by the lepton-mass corrections.

\section{KINEMATIC ANALYSIS OF HIGH-ENERGY NEUTRINO REACTIONS}

In this Section we derive Eq. (13), which gives the general form for the neutrino reaction leptonic differential cross section, $d^{2} \sigma / d \Omega_{l} d E_{l \cdot}{ }^{5}$ In particular, we find explicit expressions for the form factors $\alpha\left(q^{2}, W\right), \beta\left(q^{2}, W\right)$, and $\gamma\left(q^{2}, W\right)$, in terms of matrix elements of the vector and the axial-vector currents.

According to the effective Lagrangian of Eq. (9), the matrix element $\mathfrak{T}$ for the process $\nu+N \rightarrow l+\beta$ is given by

$$
\mathfrak{N}=g m, \quad m=\bar{u}_{l}\left(k_{l}\right) \gamma_{\lambda}\left(1+\gamma_{\tilde{5}}\right) u_{\nu}\left(k_{\nu}\right) 2^{-1 / 2}\left\langle\beta^{\text {out }}\left(k_{\beta}\right)\left|J_{\lambda}{ }^{V}+J_{\lambda}^{A}\right| N\left(k_{N}\right)\right\rangle .
$$

Here $g=\left(G \cos \theta_{C}, G \sin \theta_{C}\right)$ in $(\Delta S=0,|\Delta S|=1)$ reactions, $J_{\lambda}{ }^{V}$ and $J_{\lambda}{ }^{A}$ are the appropriate vector and axial-vector currents, and $k_{\beta}$ and $k_{N}$ are, respectively, the four-momenta of $\beta$ and of $N$. In the frame in which the initial nucleon $N$ is at rest, the reaction cross section is given by

$$
\sigma=(2 \pi)^{4} \int \frac{d^{3} k_{l}}{(2 \pi)^{3}} \int \frac{d^{3} k_{\beta}}{(2 \pi)^{3}} \sum_{\beta, \mathrm{INT}} \sum_{s} \delta\left(k_{\beta}+k_{l}-k_{\nu}-k_{N}\right)\left(\frac{m_{l}}{E_{l}} \frac{m_{\nu}}{E_{\nu}}\right) g^{2}\left\langle|m|^{2}\right\rangle .
$$

In Eq. (29), $\sum_{\beta, \text { INT }}$ is a sum over the internal variables of the system $\beta, \sum_{s}$ is an average over the initial nucleon spin, and $\left\langle|m|^{2}\right\rangle$ is the sum of $|m|^{2}$ over the lepton spin states. From Eq. (29) we get

with

$$
d^{2} \sigma / d \Omega_{l} d E_{l}=\left[g^{2} /(2 \pi)^{2}\right]\left(E_{l} / E_{v}\right)_{\kappa},
$$

$$
\kappa=\left.\sum_{\beta, \mathrm{INT}} \sum_{\delta} \delta\left(k_{\beta 0}+E_{l}-E_{\nu}-M_{N}\right) m_{l} m_{\nu}\left\langle|m|^{2}\right\rangle\right|_{\mathrm{k}_{\beta}=\mathrm{q}} .
$$

Let us now study the quantity $\kappa$. We introduce the abbreviated notation

$$
\begin{aligned}
e_{\lambda} & =2^{-1 / 2} \bar{u}_{l}\left(k_{l}\right) \gamma_{\lambda}\left(1+\gamma_{5}\right) u_{\nu}\left(k_{\nu}\right), \\
V_{\lambda}{ }^{\beta} & =\left\langle\beta^{\text {out }}\left[\mathbf{q}, i\left(q_{0}+M_{N}\right)\right]\left|J_{\lambda} V\right| N\left(\mathbf{0}, i M_{N}\right)\right\rangle, \\
A_{\lambda}{ }^{\beta} & =\left\langle\beta^{\text {out }}\left[\mathbf{q}, i\left(q_{0}+M_{N}\right)\right]\left|J_{\lambda}{ }^{A}\right| N\left(0, i M_{N}\right)\right\rangle, \\
\sum_{\beta} & =\sum_{\beta, \text { INT }} \sum_{\delta} \delta\left(k_{\beta 0}+E_{l}-E_{\nu}-M_{N}\right) .
\end{aligned}
$$

Let us further denote by $V_{D}^{\beta}$ and by $A_{D}{ }^{\beta}$ the matrix elements of the divergences of the vector and the axial-vector currents,

$$
\begin{aligned}
& V_{D^{\beta}}=-i q_{\lambda} V_{\lambda}{ }^{\beta}=\left\langle\beta^{\text {out }}\left[\mathbf{q}, i\left(q_{0}+M_{N}\right)\right]\left|\partial_{\lambda} J_{\lambda}{ }^{V}\right| N\left(\mathbf{0}, i M_{N}\right)\right\rangle, \\
& A_{D^{\beta}}=-i q_{\lambda} A_{\lambda}{ }^{\beta}=\left\langle\beta^{\text {out }}\left[\mathbf{q}, i\left(q_{0}+M_{N}\right)\right]\left|\partial_{\lambda} J_{\lambda}{ }^{A}\right| N\left(0, i M_{N}\right)\right\rangle .
\end{aligned}
$$

${ }^{5}$ Locality theorems of this type are, of course, well known. See, for example, T. D. Lee and C. N. Yang, Phys. Rev. 126, 2239 (1962); A. Pais, Phys. Rev. Letters 9, 117 (1962). 
Since the final lepton mass is neglected, we have

Using Eqs. (33) and (34), we may write

$$
q_{\lambda} e_{\lambda}=0 .
$$

$$
m=e_{\lambda}\left(V_{\lambda}^{\beta}+A_{\lambda}^{\beta}\right)=e_{n}\left(\delta_{n k}-q_{n} q_{k} / q_{0}^{2}\right)\left(V_{k}^{\beta}+A_{k}^{\beta}\right)+i\left(\mathbf{q} \cdot \mathbf{e} / q_{0}{ }^{2}\right)\left(V_{D^{\beta}}+A_{D}{ }^{\beta}\right),
$$

where the repeated indices $n$ and $k$ are summed from 1 to 3 . Defining $t_{n m}$ by

we find that

$$
t_{n m}=\left\langle e_{n} e_{m}^{*}\right\rangle m_{\nu} m_{l}=\left(k_{\nu}\right)_{n}\left(k_{l}\right)_{m}+\left(k_{l}\right)_{n}\left(k_{\nu}\right)_{m}-k_{\nu} \cdot k_{l} \delta_{n m}+\epsilon_{n m \xi \eta}\left(k_{\nu}\right)_{\xi}\left(k_{l}\right)_{\eta},
$$

$$
\begin{aligned}
& \kappa=\left.\sum_{\beta} m_{l} m_{\nu}\left\langle|m|^{2}\right\rangle\right|_{\mathbf{k}_{\beta}=\mathbf{q}} \\
& =t_{n m}\left(\delta_{m j}-q_{m} q_{j} / q_{0}^{2}\right)\left(\delta_{n k}-q_{n} q_{k} / q_{0}^{2}\right)\left\{\sum_{\beta}\left(V_{j}^{\beta}\right)^{*} V_{k}{ }^{\beta}+\sum_{\beta}\left(A_{j}{ }^{\beta}\right)^{*} A_{k}{ }^{\beta}+\sum_{\beta}\left[\left(A_{j}{ }^{\beta}\right)^{*} V_{k}{ }^{\beta}+\left(V_{j}{ }^{\beta}\right)^{*} A_{k}{ }^{\beta}\right]\right\} \\
& +t_{n m}\left(q_{n} q_{m} / q_{0}^{4}\right)\left\{\sum_{\beta}\left|V_{D^{\beta}}\right|^{2}+\sum_{\beta}\left|A_{D^{\beta}}\right|^{2}+\sum_{\beta}\left[\left(A_{D}^{\beta}\right)^{*} V_{D}{ }^{\beta}+\left(V_{D^{\beta}}\right)^{*} A_{D^{\beta}}\right]\right\} \\
& +t_{n m}\left(\delta_{n k}-q_{n} q_{k} / q_{0}^{2}\right)\left(i q_{m} / q_{0}^{2}\right)\left\{\sum_{\beta}\left[\left(V_{k}^{\beta}\right)^{*} V_{D^{\beta}}-\left(V_{D^{\beta}}\right)^{*} V_{k}^{\beta}\right]+\sum_{\beta}\left[\left(A_{k}^{\beta}\right)^{*} A_{D^{\beta}}-\left(A_{D^{\beta}}\right)^{*} A_{k^{\beta}}{ }^{\beta}\right]\right. \\
& \left.+\sum_{\beta}\left[\left(V_{k}^{\beta}\right)^{*} A_{D^{\beta}}-\left(A_{D^{\beta}}\right)^{*} V_{k}^{\beta}\right]+\sum_{\beta}\left[\left(A_{k}^{\beta}\right)^{*} V_{D^{\beta}}-\left(V_{D}^{\beta}\right)^{*} A_{k}^{\beta}\right]\right\} .
\end{aligned}
$$

The next step is to use the transformation properties of the currents under time reversal and parity to determine the form of the various $\sum_{\beta}$ terms in Eq. (37). Denoting by $T$ and by $P$ the time-reversal and parity operators, respectively, we have

$$
T J_{k}^{V}(0) T^{-1}=-J_{k}^{V}(0), \quad T J_{k}^{A}(0) T^{-1}=-J_{k}^{A}(0), \quad P J_{k}^{V}(0) P^{-1}=-J_{k}^{V}(0), \quad P J_{k}^{A}(0) P^{-1}=J_{k}^{A}(0),
$$

and similarly for the divergences of the currents. Under the assumption that the "in" and "out" states of definite total energy each form a complete basis for states of that energy, we have

$$
\sum_{\beta, \mathrm{INT}} \delta\left(k_{\beta 0}+E_{l}-E_{\nu}-M_{N}\right)\left|\beta^{\text {out }}\left(k_{\beta}\right)\right\rangle\left\langle\beta^{\text {out }}\left(k_{\beta}\right)\left|=\sum_{\beta, \mathrm{INT}} \delta\left(k_{\beta 0}+E_{l}-E_{\nu}-M_{N}\right)\right| P T \beta^{\text {out }}\left(k_{\beta}\right)\right\rangle\left\langle P T \beta^{\text {out }}\left(k_{\beta}\right)\right|,
$$

and

$$
\sum_{\boldsymbol{s}}\left|N\left(k_{N}\right)\right\rangle\left\langle N\left(k_{N}\right)\left|=\sum_{\boldsymbol{s}}\right| P T N\left(k_{N}\right)\right\rangle\left\langle P T N\left(k_{N}\right)\right|
$$

Using Eqs. (38) and (39) we find that

$$
\begin{aligned}
\sum_{\beta} V_{k}^{\beta}\left(V_{j}^{\beta}\right)^{*} & =\sum_{\beta, \mathrm{INT}} \sum_{s} \delta\left(k_{\beta 0}+E_{l}-E_{\nu}-M_{N}\right)\left\langle\beta^{\text {out }}\left|J_{k}{ }^{V}\right| N\right\rangle\left\langle\beta^{\text {out }}\left|J_{j}^{V}\right| N\right\rangle^{*} \\
& =\sum_{\beta, \text { INT }} \sum_{s} \delta\left(k_{\beta 0}+E_{l}-E_{\nu}-M_{N}\right)\left\langle P T \beta^{\text {out }}\left|J_{k} V\right| P T N\right\rangle^{*}\left\langle P T \beta^{\text {out } \mid}\left|J_{j}^{V}\right| P T N\right\rangle \\
& =\sum_{\beta} V_{j}{ }^{\beta}\left(V_{k}^{\beta}\right)^{*}=\left[\sum_{\beta} V_{k}^{\beta}\left(V_{j}^{\beta}\right)^{*}\right]^{*} .
\end{aligned}
$$

Thus, the tensor $\sum_{\beta} V_{k}^{\beta}\left(V_{j}^{\beta}\right)^{*}$ is real, and hence symmetric. Using $P$ alone shows that this tensor is an even function of q. A similar analysis can be carried through for each of the $\sum_{\beta}$ terms in Eq. (37), with the following results:

(i) $\sum_{\beta} V_{k}^{\beta}\left(V_{j}^{\beta}\right)^{*}$ and $\sum_{\beta} A_{k}^{\beta}\left(A_{j}^{\beta}\right)^{*}$ are real symmetric tensors (even under $\mathbf{q} \rightarrow-\mathbf{q}$ );

(ii) $\sum_{\beta}\left[V_{k}^{\beta}\left(A_{j}^{\beta}\right)^{*}+A_{k}{ }^{\beta}\left(V_{j}^{\beta}\right)^{*}\right]$ is an imaginary, antisymmetric pseudotensor (odd under $\mathbf{q} \rightarrow-\mathbf{q}$ );

(iii) $\sum_{\beta}\left|V_{D}^{\beta}\right|^{2}$ and $\sum_{\beta}\left|A_{D}{ }^{2}\right|^{2}$ are real scalars;

(iv) $\sum_{\beta}\left[V_{D}^{\beta}\left(A_{D}^{\beta}\right)^{*}+A_{D}^{\beta}\left(V_{D}\right)^{*}\right]$ is an imaginary pseudoscalar;

(v) $\sum_{\beta}\left[V_{k}^{\beta}\left(V_{D}^{\beta}\right)^{*}-\left(V_{k}^{\beta}\right)^{*} V_{D^{\beta}}^{\beta}\right]$ and $\sum_{\beta}\left[A_{k}^{\beta}\left(A_{D^{\beta}}\right)^{*}\right.$ $\left.-\left(A_{k}\right)^{*} A_{D}{ }^{\beta}\right]$ are imaginary vectors;

(vi) $\sum_{\beta}\left[V_{k}^{\beta}\left(A_{D}^{\beta}\right)^{*}-\left(V_{k}^{\beta}\right)^{*} A_{D}^{\beta}\right]$ and $\sum_{\beta}\left[A_{k}^{\beta}\left(V_{D}\right)^{*}\right.$ $\left.-\left(A_{k}^{\beta}\right)^{*} V_{D}^{\beta}\right]$ are imaginary pseudovectors.

All of these quantities must be formed from the one vector available, q. Thus the only possible tensors are $\delta_{k j}$ and $q_{k} q_{j}$ and the only pseudotensor is $\epsilon_{k j n} q_{n}$. No pseudovectors or pseudoscalars can be formed. Consequently, the most general from of the quantities appearing in Eq. (37) is

$$
\begin{aligned}
& \sum_{\beta}\left(V_{j}^{\beta}\right)^{*} V_{k}^{\beta}=\delta_{j k} V_{1}\left(q^{2}, W\right)+q_{j} q_{k} V_{2}\left(q^{2}, W\right), \\
& \sum_{\beta}\left(A_{j}^{\beta}\right)^{*} A_{k}^{\beta}=\delta_{j k} A_{1}\left(q^{2}, W\right)+q_{j} q_{k} A_{2}\left(q^{2}, W\right), \\
& \sum_{\beta}\left[\left(A_{j}^{\beta}\right)^{*} V_{k}^{\beta}+\left(V_{j}^{\beta}\right)^{*} A_{k^{\beta}}\right]=i \epsilon_{k j l} q_{l} I\left(q^{2}, W\right), \\
& \sum_{\beta}\left|V_{D^{\beta}}\right|^{2}=D_{V}\left(q^{2}, W\right), \\
& \sum_{\beta}\left|A_{D^{\beta}}\right|^{2}=D_{A}\left(q^{2}, W\right), \\
& \sum_{\beta}\left[\left(V_{k}^{\beta}\right)^{*} V_{D^{\beta}}-\left(V_{D^{\beta}}\right)^{*} V_{k^{\beta}}^{\beta}\right]=i q_{k} I_{V}\left(q^{2}, W\right), \\
& \sum_{\beta}\left[\left(A_{k}^{\beta}\right)^{*} A_{D^{\beta}}-\left(A_{D^{\beta}}\right)^{*} A_{k^{\beta}}^{\beta}\right]=i q_{k} I_{A}\left(q^{2}, W\right), \\
& \sum_{\beta}\left[\left(A_{D^{\beta}}^{\beta}\right)^{*} V_{D^{\beta}}+\left(V_{D^{\beta}}\right)^{*} A_{D^{\beta}}\right]=0, \\
& \sum_{\beta}\left[\left(V_{k}^{\beta}\right)^{*} A_{D^{\beta}}-\left(A_{D^{\beta}}\right)^{*} V_{k}^{\beta}\right]=0, \\
& \sum_{\beta}\left[\left(A_{k}^{\beta}\right)^{*} V_{D^{\beta}}-\left(V_{D^{\beta}}\right)^{*} A_{k^{\beta}}^{\beta}\right]=0,
\end{aligned}
$$

with all the structure functions $\left[V_{1}, V_{2}\right.$ etc. $]$ in Eq. (41) real. 
All that remains now is to evaluate the tensor contractions contained in Eq. (37). Using the equations

$$
\begin{aligned}
q_{n}\left(\delta_{n k}-q_{n} q_{k} / q_{0}^{2}\right) & =-\left(q^{2} / q_{0}^{2}\right) q_{k}, \\
q_{n} q_{m} t_{n m} & =2 E_{\nu} E_{l}\left(E_{\nu}-E_{l}\right)^{2} \cos ^{2}(\phi / 2), \\
\delta_{n m} t_{n m} & =q^{2}+2 E_{\nu} E_{l} \cos ^{2}(\phi / 2), \\
\epsilon_{n m l} q_{l} t_{n m} & =i q^{2}\left(E_{\nu}+E_{l}\right),
\end{aligned}
$$

we get, by some straightforward algebra, the result

$$
\begin{aligned}
d^{2} \sigma / d \Omega_{l} d E_{l}= & {\left[g^{2} /(2 \pi)^{2}\right]\left(E_{l} / E_{\nu}\right)_{\kappa}, } \\
\kappa= & q^{2} \alpha\left(q^{2}, W\right)+2 E_{\nu} E_{l} \cos ^{2}\left(\frac{1}{2} \phi\right) \beta\left(q^{2}, W\right) \\
& -q^{2}\left(E_{\nu}+E_{l}\right) \gamma\left(q^{2}, W\right), \\
\alpha\left(q^{2}, W\right)= & V_{1}\left(q^{2}, W\right)+A_{1}\left(q^{2}, W\right), \\
\beta\left(q^{2}, W\right)= & \left\{q^{2}\left[V_{1}\left(q^{2}, W\right)+A_{1}\left(q^{2}, W\right)\right]\right. \\
& +\left(q^{2}\right)^{2}\left[V_{2}\left(q^{2}, W\right)+A_{2}\left(q^{2}, W\right)\right] \\
& +q^{2}\left[I_{V}\left(q^{2}, W\right)+I_{A}\left(q^{2}, W\right)\right]+D_{V}\left(q^{2}, W\right) \\
& \left.+D_{A}\left(q^{2}, W\right)\right\} 4 M_{N}{ }^{2} /\left(W^{2}-M_{N}+q^{2}\right)^{2}, \\
\gamma\left(q^{2}, W\right)= & I\left(q^{2}, W\right) .
\end{aligned}
$$

The formula for antineutrino-induced reactions is the same, except that the final term in $\kappa$ is changed to $+q^{2}\left(E_{\nu}+E_{l}\right) \gamma\left(q^{2}, W\right)$ [and, of course, in Eq. (32) defining $V_{k}$ and $A_{k}$, the currents $J_{k}{ }^{V}$ and $J_{k}{ }^{A}$ are replaced by their adjoints].

The simplest illustration of our result is the elastic reaction $\nu+N \rightarrow l+N$. Explicit calculation shows that $d^{2} \sigma(\bar{\nu}+p \rightarrow \bar{l}+n) / d \Omega_{l} d E_{l}$ has the form of Eq. (13), with

$$
\begin{aligned}
& \alpha^{(-)}\left(q^{2}, W\right)=\delta\left(W-M_{N}\right)\left[\left(1+q^{2} / 4 M_{N}{ }^{2}\right) g_{A}\left(q^{2}\right)^{2}\right. \\
& \left.+\left(q^{2} / 4 M_{N}^{2}\right) g_{V}\left(q^{2}\right)^{2}\right] \\
& \beta^{(-)}\left(q^{2}, W\right)=\delta\left(W-M_{N}\right)\left[g_{A}\left(q^{2}\right)^{2}\right. \\
& \left.+F_{1}^{V}\left(q^{2}\right)^{2}+q^{2} F_{2}^{V}\left(q^{2}\right)^{2}\right] \text {, } \\
& \gamma^{(-)}\left(q^{2}, W\right)=\delta\left(W-M_{N}\right)\left[-g_{A}\left(q^{2}\right) g_{V}\left(q^{2}\right) / M_{N}\right] \text {. }
\end{aligned}
$$

We have also computed, for this reaction, the individual structure functions appearing in Eq. (41). They are

$$
\begin{gathered}
V_{1}^{(-)}\left(q^{2}, W\right)=\delta\left(W-M_{N}\right)\left(q^{2} / 4 M_{N}^{2}\right) g_{V}\left(q^{2}\right)^{2}, \\
V_{2}(-)\left(q^{2}, W\right)=\delta\left(W-M_{N}\right)\left\{\left[1+q^{2} / 4 M_{N}{ }^{2}\right] f_{V}\left(q^{2}\right)^{2}\right. \\
\left.-g_{V}\left(q^{2}\right) f_{V}\left(q^{2}\right) / M_{N}\right\}, \\
A_{1}^{(-)}\left(q^{2}, W\right)=\delta\left(W-M_{N}\right)\left(1+q^{2} / 4 M_{N}\right)^{2} g_{A}\left(q^{2}\right)^{2}, \\
A_{2}{ }^{(-)}\left(q^{2}, W\right)=\delta\left(W-M_{N}\right)\left[\left(q^{2} / 4 M_{N}^{2}\right) h_{A}\left(q^{2}\right)^{2}\right. \\
\left.-h_{A}\left(q^{2}\right) g_{A}\left(q^{2}\right) / M_{N}\right], \\
I^{(-)}\left(q^{2}, W\right)=\delta\left(W-M_{N}\right)\left[-g_{A}\left(q^{2}\right) g_{V}\left(q^{2}\right) / M_{N}\right], \\
I_{A}^{(-)}\left(q^{2}, W\right)=\delta\left(W-M_{N}\right)\left(-1 / 2 M_{N}{ }^{2}\right) \\
\times\left[2 M_{N} g_{A}\left(q^{2}\right)-q^{2} h_{A}\left(q^{2}\right)\right]^{2}, \\
D_{A}^{(-)}\left(q^{2}, W\right)=\delta\left(W-M_{N}\right)\left(q^{2} / 4 M_{N}{ }^{2}\right) \\
\times\left[2 M_{N} g_{A}\left(q^{2}\right)-q^{2} h_{A}\left(q^{2}\right)\right]^{2}, \\
I_{V}^{(-)}\left(q^{2}, W\right)=D_{V}^{(-)}\left(q^{2}, W\right)=0 .
\end{gathered}
$$

where

\section{DERIVATION OF THE SUM RULES}

In this Section we derive the sum rules of Sec. 2. In the first subsection we state and discuss the fundamental identity used in the derivations. In subsequent subsections we derive Eqs. (14), (15), and (16). The derivations for the strangeness-changing case are identical to those for the strangeness-conserving case, and are omitted.

\section{(A) Fundamental Identity}

The starting point of the derivations is the identity ${ }^{6}$

$$
\begin{aligned}
& \frac{1}{q_{0}} \int_{0}^{\infty} d t e^{i q_{0} t}\langle N|[A(t), \dot{B}(0)]| N\rangle \\
& =-i\langle N|[A(0), B(0)]| N\rangle \\
& \quad+\left(2 q_{0}\right)^{-1}\langle N|[A(0), B(0)]+[\dot{B}(0), A(0)]| N\rangle \\
& \quad+q_{0} \int_{0}^{\infty} d t e^{i q_{0} t}\langle N|[A(t), B(0)]| N\rangle,
\end{aligned}
$$

$$
\dot{A}(t)=\frac{d A(t)}{d t}, \quad \dot{B}(t)=\frac{d B(t)}{d t}
$$

are the time derivatives of $A(t)$ and $B(t)$. Equation (46) is easily derived by repeated integration by parts, and holds for all $q_{0}$ in the upper half of the complex plane. In this paper, the operators $A(t)$ and $B(t)$ will always be of the form

$$
\begin{gathered}
A(t)=-i \int d^{3} x e^{-i \mathbf{s} \cdot \mathbf{x}} \mathfrak{F}_{A}(\mathbf{x}, t) \\
B(t)=-i \int d^{3} y e^{i \mathbf{s} \cdot \mathbf{y}} \mathfrak{F}_{B}(\mathbf{y}, t) ; \\
\mathfrak{F}_{A}=\mathfrak{F}_{a \lambda} \text { or } \mathfrak{F}_{a \lambda}{ }^{5}, \quad \mathfrak{F}_{B}=\mathfrak{F}_{b \sigma} \text { or } \mathfrak{F}_{b \sigma}{ }^{5}
\end{gathered}
$$

In (I) we studied Eq. (46) with $s=0$; this led, in the limit $q_{0} \rightarrow 0$, to sum rules at $q^{2}=0$. In this paper we will study the case when $\mathbf{s} \neq 0$, and will find, in the limit as $q_{0} \rightarrow 0$, sum rules for fixed $q^{2}$ (with $q^{2}=|\mathbf{s}|^{2}$ ).

There are a number of features which all of the derivations given below have in common. First of all, we will always use Eq. (46) with the nucleon $N$ at rest, and with the nucleon spin averaged over. Secondly, each term of Eq. (46) can be divided into a part which is symmetric and a part which is antisymmetric in the unitary spin indices $a$ and $b$. We will only study the identity for the antisymmetric parts. In each case below, we will find that the term

$$
U=\left(2 q_{0}\right)^{-1}\langle N|[\dot{A}(0), B(0)]+[\dot{B}(0), A(0)]| N\rangle
$$

- Equation (46) is a more symmetrical version of Eq. (37) of Ref. 2. Equation (46) remains valid if $\langle N|$ and $|N\rangle$ are replaced by any two states of equal four-momentum. 
is purely symmetric in the unitary spin indices, and thus makes no contribution. Thirdly, since we have

$$
\begin{aligned}
& q_{0} \int_{0}^{\infty} d t e^{i q_{0} t}\langle N|[A(t), B(0)]| N\rangle \\
&=-i q_{0} \sum_{\substack{\beta, \mathrm{INT} \\
\left(M_{\beta} \geq M_{N}\right)}}\left\{\frac{\left\langle N\left|\Im_{A}\right| \beta\right\rangle\left\langle\beta\left|F_{B}\right| N\right\rangle}{q_{0}+M_{N}-\left(|\mathbf{s}|^{2}+M_{\beta}^{2}\right)^{1 / 2}}\right. \\
&\left.\quad-\frac{\left\langle N\left|\mathfrak{F}_{B}\right| \beta\right\rangle\left\langle\beta\left|\Im_{A}\right| N\right\rangle}{q_{0}+\left(|\mathbf{s}|^{2}+M_{\beta}\right)^{1 / 2}-M_{N}}\right\}(2 \pi)^{3} \delta(\mathbf{0}),
\end{aligned}
$$

the limit as $q_{0} \rightarrow 0$ of Eq. (50) is zero for all $|\mathbf{s}|^{2}>0$. As a result, the third term on the right-hand side of Eq. (46) makes no contribution to the sum rules. ${ }^{7}$

Finally, we will always find that the unitary spinantisymmetric part of

$$
\int_{0}^{\infty} d t e^{i q_{0} t}\langle N|[A(t), \dot{B}(0)]| N\rangle
$$

is an odd function of $q_{0}, O\left(q_{0}, q^{2}\right)$. Thus, in the limit as $q_{0} \rightarrow 0$ the identity of Eq. (46) will become the equation

$$
\left.\frac{\partial}{\partial q_{0}} O\left(q_{0}, q^{2}\right)\right|_{q_{0}=0}=C,
$$

where $C$ is the unitary spin-antisymmetric part of the commutator $-i\langle N|[A(0), B(0)]| N\rangle$. Equation (52) states that the commutator of $A$ and $B$ is related to the energy derivative of a forward scattering amplitude, evaluated at zero energy. Up to this point the derivation is rigorous. Now, in order to relate the left-hand side of Eq. (52) to physically measurable quantities, we will assume that the energy derivative $\left(\partial / \partial q_{0}\right) O\left(q_{0}, q^{2}\right)$ satisfies an unsubtracted dispersion relation in the energy variable $q_{0}$, for fixed $q^{2}$. The discontinuity of $\left(\partial / \partial q_{0}\right)$ $\times O\left(q_{0}, q^{2}\right)$ across its cuts will, in each case considered, be related to the structure functions defined in Eq. (41).

\section{(B) Sum Rule for $\boldsymbol{\beta}^{( \pm)}$}

The sum rule on $\beta^{( \pm)}$of Eq. (14) is obtained by adding together two separately derived sum rules on the axialvector and the vector parts of $\beta^{( \pm)}, \beta_{A}{ }^{( \pm)}$, and $\beta_{V}^{( \pm)}$:

$$
\begin{aligned}
1=g_{A}\left(q^{2}\right)^{2}+\int_{M_{N}+M_{\pi}}^{\infty} & \frac{W}{M_{N}} d W \\
& \times\left[\beta_{A}{ }^{(-)}\left(q^{2}, W\right)-\beta_{A}^{(+)}\left(q^{2}, W\right)\right],
\end{aligned}
$$

$$
\begin{aligned}
1=F_{1} V\left(q^{2}\right)^{2}+q^{2} F_{2} V\left(q^{2}\right)^{2}+\int_{M_{N}+M_{\pi}}^{\infty} \frac{W}{M_{N}} d W \\
\times\left[\beta_{V}^{(-)}\left(q^{2}, W\right)-\beta_{V}{ }^{(+)}\left(q^{2}, W\right)\right] .
\end{aligned}
$$

In terms of the structure functions defined in Eq. (41),

$$
\begin{array}{r}
\beta_{A}{ }^{( \pm)}\left(q^{2}, W\right)=\left[q^{2} A_{1}^{( \pm)}\left(q^{2}, W\right)+\left(q^{2}\right)^{2} A_{2}{ }^{( \pm)}\left(q^{2}, W\right)\right. \\
\left.+q^{2} I_{A}{ }^{( \pm)}\left(q^{2}, W\right)+D_{A}{ }^{( \pm)}\left(q^{2}, W\right)\right] \\
\times 4 M_{N} 2 /\left(W^{2}-M_{N}{ }^{2}+q^{2}\right)^{2}, \\
\beta_{V}{ }^{( \pm)}\left(q^{2}, W\right)=q^{2}\left[V_{1}^{( \pm)}\left(q^{2}, W\right)+q^{2} V_{2}{ }^{( \pm)}\left(q^{2}, W\right)\right] \\
\times 4 M_{N} /\left(W^{2}-M_{N}{ }^{2}+q^{2}\right)^{2} .
\end{array}
$$

[The structure functions $I_{V}{ }^{( \pm)}\left(q^{2}, W\right)$ and $D_{V}{ }^{( \pm)}\left(q^{2}, W\right)$ vanish identically in the strangeness-conserving case, because of conservation of the vector current.] Since the derivations of Eqs. (53a) and (53b) are identical, we will treat explicitly only the axial-vector case, Eq. (53a).

We start from the fundamental identity of Eq. (46), taking

$$
\begin{aligned}
& A(t)=-i \int d^{3} x e^{-i \mathbf{s} \cdot \mathbf{x}} \mathfrak{F}_{a 4^{5}}(\mathbf{x}, t), \\
& B(t)=-i \int d^{3} y e^{i \mathbf{s} \cdot \mathbf{y}} \mathfrak{F}_{b 4^{5}}(\mathbf{y}, t) .
\end{aligned}
$$

Defining $D_{a}(x)=\partial_{\lambda} \mathfrak{F}_{a \lambda^{5}}(x)$ we find, by spatial integration by parts, that

$$
\begin{aligned}
& A(t)=\int d^{3} x e^{-i \mathrm{~s} \cdot \mathbf{x}}\left[D_{a}(\mathbf{x}, t)-i s_{n} \mathscr{F}_{a n}{ }^{5}(\mathbf{x}, t)\right], \\
& \dot{B}(t)=\int d^{3} y e^{i \mathbf{s} \cdot \mathbf{y}}\left[D_{b}(\mathbf{y}, t)+i s_{n} \mathscr{F}_{b n}{ }^{5}(\mathbf{y}, t)\right],
\end{aligned}
$$

where the repeated index $n$ is summed over. With $A$ and $B$ as shown in Eq. (55), the first term on the right-hand side of Eq. (46) becomes, using the local commutation relation of Eq. (1c),

$$
-i \sum_{s}\langle N|[A(0), B(0)]| N\rangle=\boldsymbol{\epsilon}_{a b c}\left\langle\frac{1}{2} \tau^{c}\right\rangle(2 \pi)^{3} \delta(\mathbf{0}) .
$$

Thus this term is purely antisymmetric in the isospin indices $a$ and $b$. [Note that the validity of Eq. (57) depends on the correctness of the local commutation relation. If Eq. (1c) were modified by the addition of a term proportional to $\nabla^{2} \delta(\mathbf{x}-\mathbf{y})$, a term proportional to $|\mathbf{s}|^{2}$ would be added to Eq. (57).] The second term on the right-hand side of Eq. (46) becomes

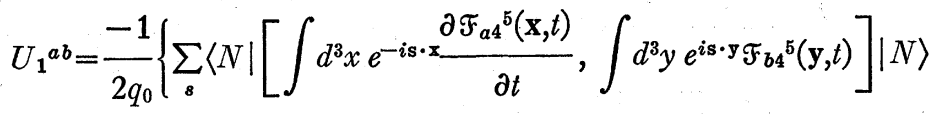

$$
\begin{aligned}
& \left.+\sum_{\boldsymbol{s}}\left\langle N\left|\left[\int d^{3} y e^{i \mathbf{s} \cdot y} \frac{\partial \mathscr{F}_{b 4^{5}(y, t)}}{\partial t}, \int d^{3} x e^{-i \mathbf{s} \cdot \mathbf{x} \mathfrak{F}_{a 4}{ }^{5}(\mathbf{x}, t)}\right]\right| N\right\rangle\right\}
\end{aligned}
$$

${ }^{7}$ Only when $|\mathbf{s}|^{2}=0$ does the one-nucleon intermediate state $\left(M_{\beta}=M_{N}\right)$ make a contribution to the limit. This is the case considered in Ref. 2. 


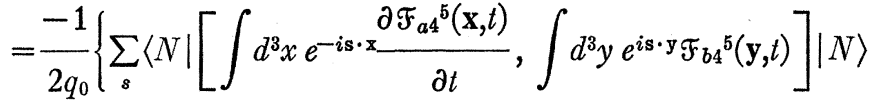

$$
\begin{aligned}
& \left.+\sum_{\boldsymbol{s}}\left\langle N\left|\left[\int d^{3} x e^{-i \mathrm{~s} \cdot \mathbf{x}} \frac{\partial \mathfrak{F}_{b 4}{ }^{5}(\mathbf{x}, t)}{\partial t}, \int d^{3} y e^{i \mathbf{s} \cdot \mathbf{y}} \mathfrak{F}_{a 4}{ }^{5}(\mathbf{y}, t)\right]\right| N\right\rangle\right\},
\end{aligned}
$$

where we have obtained Eq. (58b) by setting $-\mathbf{y} \leftrightarrow \mathbf{x}$ in the second term of Eq. (58a) and by using the parity transformation properties of the axial-vector current. Clearly $U_{1}{ }^{a b}$ is explicitly symmetric in $a$ and $b$. Thus, if we agree to keep only the part of Eq. (46) which is antisymmetric in $a$ and $b$, the second term on the right-hand side of Eq. (46), which involves the unknown commutator of $\partial \mathfrak{F}_{a 4}{ }^{5} / \partial t$ with $\mathfrak{F}_{b 4}{ }^{5}$, drops out. As discussed above, the limit as $q_{0} \rightarrow 0$ of the third term on the right-hand side of Eq. (46) vanishes.

Now let us turn to the left-hand side of Eq. (46). Using translational invariance, the integral over y can be done explicitly, giving an over-all factor of $(2 \pi)^{3} \delta(0)$. We cancel this against the identical factor in Eq. (57). Taking $N$ to be a proton at rest, and multiplying Eq. (46) by an over-all factor $\epsilon_{a b 3}$ gives

$$
\begin{gathered}
1=\frac{\epsilon_{a b 3}}{q_{0}} \int d^{4} x \exp (-i \hat{q} \cdot x) \theta\left(x_{0}\right) \sum_{s}\left\langle p\left|\left[D_{a}(x)-i s_{n} F_{a n}{ }^{5}(x), D_{b}(0)+i s_{m} F_{b m}{ }^{5}(0)\right]\right| p\right\rangle+o\left(q_{0}\right), \\
\hat{q}=\left(\mathbf{s}, i q_{0}\right),
\end{gathered}
$$

where $o\left(q_{0}\right)$ indicates terms which vanish as $q_{0} \rightarrow 0$. Let us define the amplitudes $d\left(q_{0}, q^{2}\right), a_{1}\left(q_{0}, q^{2}\right), a_{2}\left(q_{0}, q^{2}\right)$, and $i_{A}\left(q_{0}, q^{2}\right)$ by the equations

$$
\begin{gathered}
d\left(q_{0}, q^{2}\right)=\epsilon_{a b 3} \int d^{4} x e^{-i q \cdot x} \theta\left(x_{0}\right) \sum_{s}\left\langle p\left|\left[D_{a}(x), D_{b}(0)\right]\right| p\right\rangle, \\
a_{1}\left(q_{0}, q^{2}\right) \delta_{n m}+a_{2}\left(q_{0}, q^{2}\right) q_{n} q_{m}=\epsilon_{a b 3} \int d^{4} x e^{-i q \cdot x} \theta\left(x_{0}\right) \sum_{s}\left\langle p\left|\left[\mathcal{F}_{a n}{ }^{5}(x), \mathcal{F}_{b m}{ }^{5}(0)\right]\right| p\right\rangle, \\
i q_{n} i_{A}\left(q_{0}, q^{2}\right)=\epsilon_{a b 3} \int d^{4} x e^{-i q \cdot x} \theta\left(x_{0}\right) \sum_{s}\left\langle p\left|\left[\mathscr{F}_{a n}{ }^{5}(x), D_{b}(0)\right]-\left[D_{a}(x), \mathcal{F}_{b n}{ }^{5}(0)\right]\right| p\right\rangle .
\end{gathered}
$$

We will prove below that these are all odd functions of $q_{0}$. Thus Eq. (59), in the limit as $q_{0} \rightarrow 0$, becomes the statement

$$
\begin{aligned}
1 & =\left.\frac{\partial}{\partial q_{0}} \lambda\left(q_{0}, q^{2}\right)\right|_{q_{0}=0}, \\
\lambda\left(q_{0}, q^{2}\right) & =d\left(q_{0}, q^{2}\right)+q^{2} a_{1}\left(q_{0}, q^{2}\right)+\left(q^{2}\right)^{2} a_{2}\left(q_{0}, q^{2}\right)+q^{2} i_{A}\left(q_{0}, q^{2}\right),
\end{aligned}
$$

with $q^{2}$ fixed at $|\mathbf{s}|^{2}$.

Let us now study the properties of the functions $d, a_{1}, a_{2}$, and $i_{A}$. From their definitions as retarded commutators, it follows by the standard methods of forward dispersion relations ${ }^{8}$ that they are analytic functions of $q_{0}$ in the upper half $q_{0}$ plane, for fixed $q^{2}$. Thus if we assume that the amplitude $\left(\partial / \partial q_{0}\right) \lambda\left(q_{0}, q^{2}\right)$ approaches zero as $q_{0} \rightarrow \infty$ in the upper half plane, we can write the unsubtracted dispersion relation

$$
\frac{\partial}{\partial q_{0}} \lambda\left(q_{0}, q^{2}\right)=\frac{1}{\pi} \int_{-\infty}^{\infty} \frac{d q_{0}{ }^{\prime}}{\left(q_{0}{ }^{\prime}-q_{0}\right)^{2}}\left\{d^{\prime}\left(q_{0}{ }^{\prime}, q^{2}\right)+q^{2} a_{1}{ }^{\prime}\left(q_{0}{ }^{\prime}, q^{2}\right)+\left(q^{2}\right)^{2} a_{2}{ }^{\prime}\left(q_{0}{ }^{\prime}, q^{2}\right)+q^{2} i_{A}{ }^{\prime}\left(q_{0}{ }^{\prime}, q^{2}\right)\right\},
$$

where the absorptive parts $d^{\prime}, a_{1}{ }^{\prime}, a_{2}{ }^{\prime}, i_{A}{ }^{\prime}$ are defined by

$$
\begin{gathered}
i d^{\prime}\left(q_{0}, q^{2}\right)=\frac{1}{2} \epsilon_{a b 3} \int d^{4} x e^{-i q \cdot x} \sum_{s}\left\langle p\left|\left[D_{a}(x), D_{b}(0)\right]\right| p\right\rangle, \\
i\left[a_{1}^{\prime}\left(q_{0}, q^{2}\right) \delta_{n m}+a_{2}{ }^{\prime}\left(q_{0}, q^{2}\right) q_{n} q_{m}\right]=\frac{1}{2} \epsilon_{a b 3} \int d^{4} x e^{-i q \cdot x} \sum_{s}\left\langle p\left|\left[\mathscr{F}_{a n}{ }^{5}(x), F_{b m}{ }^{5}(0)\right]\right| p\right\rangle, \\
i\left[i q_{n} i_{A}{ }^{\prime}\left(q_{0}, q^{2}\right)\right]=\frac{1}{2} \epsilon_{a b 3} \int d^{4} x e^{-i q \cdot x} \sum_{s}\left\langle p\left|\left[\mathscr{F}_{a n}{ }^{5}(x), D_{b}(0)\right]-\left[D_{a}(x), \mathcal{F}_{b n}{ }^{5}(0)\right]\right| p\right\rangle .
\end{gathered}
$$

${ }^{8}$ J. D. Jackson, Dispersion Relations, edited by G. R. Screaton (Interscience Publishers, Inc., New York, 1961), pp. 1-32. 
The next step is to evaluate the absorptive parts. Let us consider explicitly the case of $d^{\prime}$. Let $k_{p}=\left(0, i M_{N}\right)$ be the proton four-momentum. Inserting a complete set of intermediate states, we find that

$$
\begin{aligned}
i d^{\prime}\left(q_{0}, q^{2}\right)= & \frac{1}{2} \epsilon_{a b 3}(2 \pi)^{4} \sum_{\beta, \mathrm{INT}} \sum_{s} \int \frac{d^{3} k_{\beta}}{(2 \pi)^{3}} \\
& \times\left[\left\langle p\left|D_{a}(0)\right| \beta\left(k_{\beta}\right)\right\rangle\left\langle\beta\left(k_{\beta}\right)\left|D_{b}(0)\right| p\right\rangle \delta\left(k_{\beta}-q-k_{p}\right)-\left\langle p\left|D_{b}(0)\right| \beta\left(k_{\beta}\right)\right\rangle\left\langle\beta\left(k_{\beta}\right)\left|D_{a}(0)\right| p\right\rangle \delta\left(k_{\beta}+q-k_{p}\right)\right] \\
= & \pi \epsilon_{a b 3} \sum_{\beta, \mathrm{INT}} \sum_{\delta}\left\{\left.\left[\left\langle p\left|D_{a}(0)\right| \beta\left(k_{\beta}\right)\right\rangle\left\langle\beta\left(k_{\beta}\right)\left|D_{b}(0)\right| p\right\rangle\right]\right|_{\mathrm{k}_{\beta}=\mathrm{q}} \delta\left(k_{\beta 0}-q_{0}-M_{N}\right)\right. \\
& \left.-\left.\left[\left\langle p\left|D_{b}(0)\right| \beta\left(k_{\beta}\right)\right\rangle\left\langle\beta\left(k_{\beta}\right)\left|D_{a}(0)\right| p\right\rangle\right]\right|_{\mathrm{k}_{\beta}=-\mathrm{q}} \delta\left(k_{\beta 0}+q_{0}-M_{N}\right)\right\} .
\end{aligned}
$$

Parity invariance tells us that

$$
\begin{aligned}
\left.\sum_{\beta, \mathrm{INT}} \sum_{\boldsymbol{s}}\left[\left\langle p\left|D_{b}(0)\right| \beta\left(k_{\beta}\right)\right\rangle\left\langle\beta\left(k_{\beta}\right)\left|D_{a}(0)\right| p\right\rangle\right]\right|_{\mathrm{k}_{\beta}=-\mathrm{q}} \delta\left(k_{\beta 0}+q_{0}-M_{N}\right) \\
\quad=\left.\sum_{\beta, \mathrm{INT}} \sum_{\boldsymbol{s}}\left[\left\langle p\left|D_{b}(0)\right| \beta\left(k_{\beta}\right)\right\rangle\left\langle\beta\left(k_{\beta}\right)\left|D_{a}(0)\right| p\right\rangle\right]\right|_{\mathrm{k}_{\beta}=q} \delta\left(k_{\beta 0}+q_{0}-M_{N}\right) .
\end{aligned}
$$

Thus Eq. (64) can be written, using the antisymmetry of $\epsilon_{a b 3}$, as

$$
i d^{\prime}\left(q_{0}, q^{2}\right)=\left.\pi \epsilon_{a b 3} \sum_{\beta, \mathrm{INT}} \sum_{s}\left[\left\langle p\left|D_{a}(0)\right| \beta\left(k_{\beta}\right)\right\rangle\left\langle\beta\left(k_{\beta}\right)\left|D_{b}(0)\right| p\right\rangle\right]\right|_{\mathrm{k}_{\beta}=q}\left[\delta\left(k_{\beta 0}-q_{0}-M_{N}\right)+\delta\left(k_{\beta 0}+q_{0}-M_{N}\right)\right] .
$$

We see that $d^{\prime}$ is an even function of $q_{0}$; hence $d$ is an odd function of $q_{0}$. Since

$$
\epsilon_{a b 3} D_{a}{ }^{*} D_{b}=D_{1}^{*} D_{2}-D_{2}^{*} D_{1}=\frac{1}{2} i\left[\left(D_{1}^{*}+i D_{2}^{*}\right)\left(D_{1}-i D_{2}\right)-\left(D_{1}^{*}-i D_{2}^{*}\right)\left(D_{1}+i D_{2}\right)\right],
$$

we obtain finally the result that

with

$$
d^{\prime}\left(q_{0}, q^{2}\right)=\frac{1}{2} \pi\left[D^{(-)}-D^{(+)}\right], \quad q_{0}>0,
$$

$$
\begin{aligned}
& D^{(-)}=\sum_{\beta, \mathrm{INT}} \sum_{s}\left|\left\langle\beta\left[\mathbf{q}, i\left(q_{0}+M_{N}\right)\right]\left|D_{1}(0)-i D_{2}(0)\right| p\right\rangle\right|^{2} \delta\left(k_{\beta 0}-q_{0}-M_{N}\right), \\
& D^{(+)}=\sum_{\beta, \mathrm{INT}} \sum_{s}\left|\left\langle\beta\left[\mathbf{q}, i\left(q_{0}+M_{N}\right)\right]\left|D_{1}(0)+i D_{2}(0)\right| p\right\rangle\right|^{2} \delta\left(k_{\beta 0}-q_{0}-M_{N}\right) .
\end{aligned}
$$

Clearly Eq. (69) is identical with Eqs. (41), (32), and (33), defining the structure function $D$, with $q_{0}$ given by

$$
q_{0}=E_{\nu}-E_{l}=\left(W^{2}-M_{N}^{2}+q^{2}\right) / 2 M_{N} .
$$

In a similar manner we find that $a_{1}{ }^{\prime}, a_{2}{ }^{\prime}$, and $i_{A}{ }^{\prime}$ are even functions of $q_{0}$ (which implies that $a_{1}, a_{2}$, and $i_{A}$ are odd functions of $\left.q_{0}\right)$. Also, we find that for $q_{0}>0$,

$$
a_{1}{ }^{\prime}\left(q_{0}, q^{2}\right)=\frac{1}{2} \pi\left[A_{1}^{(-)}-A_{1}^{(+)}\right], \quad a_{2}{ }^{\prime}\left(q_{0}, q^{2}\right)=\frac{1}{2} \pi\left[A_{2}^{(-)}-A_{2}^{(+)}\right], \quad i_{A}{ }^{\prime}\left(q_{0}, q^{2}\right)=\frac{1}{2} \pi\left[I_{A}^{(-)}-I_{A}^{(+)}\right],
$$

where the structure functions $A_{1}{ }^{( \pm)}, A_{2}{ }^{( \pm)}$, and $I_{A}{ }^{( \pm)}$are those defined in Eq. (41). Combining Eqs. (43), (61), (62), and (68)-(71), we see that we have derived the sum rule

$$
1=\int d q_{0}\left[\beta_{A}^{(-)}-\beta_{A}^{(+)}\right]=\int \frac{W}{M_{N}} d W\left[\beta_{A}^{(-)}\left(q^{2}, W\right)-\beta_{A}^{(+)}\left(q^{2}, W\right)\right] .
$$

Using Eq. (44), the pole contribution to Eq. (72) can be explicitly evaluated, giving Eq. (53a).

\section{(C) Sum Rule for $\boldsymbol{\alpha}^{( \pm)}$}

The sum rule on $\alpha^{( \pm)}$of Eq. (15) is obtained by adding together the two identities

$$
\begin{aligned}
C_{I}^{2} & =\left(1+\frac{q^{2}}{4 M_{N}{ }^{2}}\right) g_{A}\left(q^{2}\right)^{2}+\int_{M_{N}+M_{\pi}}^{\infty} \frac{W}{M_{N}} d W\left[\alpha_{A}{ }^{(-)}\left(q^{2}, W\right)-\alpha_{A}^{(+)}\left(q^{2}, W\right)\right], \\
C_{I}{ }^{1} & =\left(\frac{q^{2}}{4 M_{N}{ }^{2}}\right) g_{V}\left(q^{2}\right)^{2}+\int_{M_{N}+M_{\pi}}^{\infty} \frac{W}{M_{N}} d W\left[\alpha_{V}{ }^{(-)}\left(q^{2}, W\right)-\alpha_{V}^{(+)}\left(q^{2}, W\right)\right] .
\end{aligned}
$$

Here $\alpha_{A}{ }^{( \pm)}$and $\alpha_{V}{ }^{( \pm)}$are, respectively, the axial-vector and the vector parts of $\alpha^{( \pm)}$,

$$
\alpha_{A}^{( \pm)}=A_{1}^{( \pm)}\left(q^{2}, W\right), \quad \alpha_{V}^{( \pm)}=V_{1}^{( \pm)}\left(q^{2}, W\right) .
$$


We will sketch the derivation of Eq. (73a); the derivation of Eq. (73b) is identical.

In order to derive Eq. (73a), we use the fundamental identity, with

$$
A(t)=-i \int d^{3} x e^{-i s \cdot x} \mathfrak{F}_{a n}{ }^{5}(\mathbf{x}, t), \quad B(t)=-i \int d^{3} y e^{i \mathbf{s} \cdot \mathbf{y}} \mathfrak{F}_{b m^{5}}(\mathbf{y}, t) .
$$

Using Eqs. (4a) and (11), the first term on the right-hand side of Eq. (46) becomes

$$
-i \sum_{s}\langle p|[A(0), B(0)]| p\rangle=-\epsilon_{a b c} \delta_{n m} C_{I}^{2}\left\langle\frac{1}{2} \tau^{c}\right\rangle(2 \pi)^{3} \delta(\mathbf{0})+(\text { symmetric in } a b) .
$$

The second term is

$$
U_{2}{ }^{n m, a b}=\frac{-1}{2 q_{0}} \sum_{s}\left\langle p\left|\int d^{3} x e^{-i \mathbf{s} \cdot \mathbf{x}} \int d^{3} y e^{i s \cdot \mathbf{y}}\left\{\left[\frac{\partial \mathscr{F}_{a n^{5}}{ }^{5}(\mathbf{x}, t)}{\partial t}, \mathscr{F}_{b m^{5}}{ }^{5}(\mathbf{y}, t)\right]+\left[\frac{\partial \mathscr{F}_{b m}{ }^{5}(\mathbf{y}, t)}{\partial t}, \mathscr{F}_{a n}{ }^{5}(\mathbf{x}, t)\right]\right\}\right| p\right\rangle,
$$

which, by using the parity transformation properties of $\mathfrak{F}^{5}$, is equal to

$$
\frac{-1}{2 q_{0}} \sum_{s}\left\langle p\left|\int d^{3} x e^{-i \mathbf{s} \cdot \mathbf{x}} \int d^{3} y e^{i \mathbf{s} \cdot \mathbf{v}}\left\{\left[\frac{\partial \mathscr{F}_{a n}{ }^{5}(\mathbf{x}, t)}{\partial t}, \mathfrak{F}_{b m^{5}}{ }^{5}(\mathbf{y}, t)\right]+\left[\frac{\partial \mathfrak{F}_{b m^{5}}(\mathbf{x}, t)}{\partial t}, \mathfrak{F}_{a n^{5}}(\mathbf{y}, t)\right]\right\}\right| p\right\rangle .
$$

The expression in Eq. (78) is explicitly symmetric under the simultaneous interchanges $n \leftrightarrow m, a \leftrightarrow b$. Since parity invariance requires that $U_{2}$ be of the form

$$
U_{2}^{n m, a b}=\mu_{1}^{a b} \delta_{n m}+\mu_{2}^{a b} s_{n} s_{m},
$$

$U_{2}$ is symmetric under the interchange $a \leftrightarrow b$. Thus, if we keep only terms which are antisymmetric in $a$ and $b$, the unwanted $\left[\partial \mathcal{F}^{5} / \partial t, \mathfrak{F}^{5}\right]$ terms drop out.

As a result, we are left with the identity

$$
\begin{aligned}
& \delta_{n m} C_{I}^{2}=\left.\frac{\partial}{\partial q_{0}} \eta\left(q_{0}, q^{2}\right)\right|_{q_{0}=0}, \\
& \eta\left(q_{0}, q^{2}\right)=\bar{a}_{1}\left(q_{0}, q^{2}\right) \delta_{n m}+\bar{a}_{2}\left(q_{0}, q^{2}\right) q_{n} q_{m}=\epsilon_{a b 3} \int d^{4} x e^{-i q \cdot x} \theta\left(x_{0}\right) \sum_{s}\left\langle p\left|\left[\frac{\partial \mathcal{F}_{a n}{ }^{5}(x)}{\partial x_{0}}, \frac{\partial \mathcal{F}_{b m^{5}}{ }^{5}(0)}{\partial t}\right]\right| p\right\rangle .
\end{aligned}
$$

[Here $\partial \mathscr{F}_{b m}{ }^{5}(0) / \partial t$ denotes $\partial \mathscr{F}_{b m}{ }^{5}(\mathbf{y}, t) / \partial t$ evaluated at $\left.\mathbf{y}=\mathbf{0}, t=0.\right]$ Let us now postulate that

$$
\partial \bar{a}_{1}\left(q_{0}, q^{2}\right) / \partial q_{0}
$$

satisfies an unsubtracted dispersion relation. It is easy to see that the absorptive part of $\bar{a}_{1}\left(q_{0}, q^{2}\right)$ is just $q_{0}^{2}$ times the absorptive part of the amplitude $a_{1}\left(q_{0}, q^{2}\right)$ defined in Eq. (60). Thus, the $\delta_{n m}$ term in Eq. (80) becomes

$$
C_{I}{ }^{2}=\int d q_{0}\left(A_{1}^{(-)}-A_{1}^{(+)}\right)=\int \frac{W}{M_{N}} d W\left[A_{1}^{(-)}\left(q^{2}, W\right)-A_{1}^{(+)}\left(q^{2}, W\right)\right],
$$

which is the result to be proved.

\section{(D) Sum Rule for $\boldsymbol{\gamma}^{( \pm)}$}

The sum rule on $\gamma^{( \pm)}$of Eq. (16) is derived by adding the fundamental identity, with

$$
A_{\mathbf{1}}(t)=-i \int d^{3} x e^{-i \mathbf{s} \cdot \mathbf{x}} \mathfrak{F}_{a n}{ }^{5}(\mathbf{x}, t), \quad B_{1}(t)=-i \int d^{3} y e^{i \mathbf{s} \cdot \mathbf{y}} \mathfrak{F}_{b m}(\mathbf{y}, t),
$$

to the same identity, with

$$
A_{2}(t)=-i \int d^{3} x e^{-i \mathbf{s} \cdot \mathbf{x}} \mathfrak{F}_{a n}(\mathbf{x}, t), \quad B_{2}(t)=-i \int d^{3} y e^{i \mathbf{s} \cdot \mathbf{y}} \mathfrak{F}_{b m}{ }^{5}(\mathbf{y}, t)
$$

Using Eq. (4b), the first term on the right-hand side of Eq. (46) is

$$
-i \sum_{\boldsymbol{s}}\left\langle p\left|\left[A_{1}(0), B_{1}(0)\right]+\left[A_{2}(0), B_{2}(0)\right]\right| p\right\rangle=(\text { symmetric in } a b),
$$


since

$$
\sum_{s}\left\langle p\left|Q_{c 4}(0)\right| p\right\rangle=0
$$

for nucleon states at rest. The second term, using the parity transformation properties of the currents, becomes

$$
\begin{aligned}
& U_{3}{ }^{n m, a b}=\frac{-1}{2 q_{0}} \sum_{\boldsymbol{s}}\langle p| \int d^{3} x e^{-i \mathbf{s} \cdot \mathbf{x}} \int d^{3} y e^{i \mathbf{s} \cdot \mathbf{y}}\left\{\left[\frac{\partial \mathcal{F}_{a n}{ }^{5}(\mathbf{x}, t)}{\partial t}, \mathfrak{F}_{b m}(\mathbf{y}, t)\right]-\left[\frac{\partial \mathcal{F}_{b m}(\mathbf{x}, t)}{\partial t}, \mathscr{F}_{a n}{ }^{5}(\mathbf{y}, t)\right]\right. \\
& \left.+\left[\frac{\partial \mathscr{F}_{a n}(\mathbf{x}, t)}{\partial t}, \mathfrak{F}_{b m}{ }^{5}(\mathbf{y}, t)\right]-\left[\frac{\partial \mathscr{F}_{b m}{ }^{5}(\mathbf{x}, t)}{\partial t}, \mathscr{F}_{a n}(\mathbf{y}, t)\right]\right\}|p\rangle \\
& =\mu_{3}{ }^{a b} \epsilon_{n m l} s_{l} .
\end{aligned}
$$

Clearly, $\mu_{3}{ }^{a b}$ is symmetric in $a$ and $b$. If we keep only the antisymmetric part of the identity, the $\left[\partial \mathcal{F}^{5} / \partial t, \mathfrak{F}\right]$ and the $\left[\partial \mathscr{F} / \partial t, \mathfrak{F}^{5}\right]$ terms drop out.

Thus, we get the identity

$$
\begin{aligned}
& 0=\left.\frac{\partial}{\partial q_{0}} i\left(q_{0}, q^{2}\right)\right|_{q_{0}=0} \\
& i\left(q_{0}, q^{2}\right)=\epsilon_{a b 3} \int d^{4} x e^{-i q \cdot x} \theta\left(x_{0}\right) \sum_{s}\left\langle p\left|\left[\frac{\partial \mathcal{F}_{a n}{ }^{5}(x)}{\partial x_{0}}, \frac{\partial \mathscr{F}_{b m}(0)}{\partial t}\right]+\left[\frac{\partial \mathcal{F}_{a n}(x)}{\partial x_{0}}, \frac{\partial \mathscr{F}_{b m}{ }^{5}(0)}{\partial t}\right]\right| p\right\rangle .
\end{aligned}
$$

The postulate that

$$
\partial i\left(q_{0}, q^{2}\right) / \partial q_{0}
$$

satisfies an unsubtracted dispersion relation in $q_{0}$ leads immediately to Eq. (16).

\section{ACKNOWLEDGMENTS}

I am grateful to Professor M. Gell-Mann for a discussion essential to the genesis of this paper, and to Dr. C. G. Callan for an interesting conversation. I wish to thank Professor L. Van Hove for the hospitality of the CERN Theoretical Study Division during the summer of 1965.

\section{APPENDIX}

In this Appendix we give the generalization of the results stated in Sec. 2 to the case when all lepton-mass terms are included. In order to calculate lepton-mass corrections, it is easier to work covariantly, rather than to eliminate the fourth components of currents in terms of spatial components and divergences. Thus we write

$$
\begin{aligned}
T_{\lambda \sigma} & =\sum_{\beta, 1 \mathrm{NT}} \sum_{\delta} \delta\left(k_{\beta 0}-k_{N 0}-q_{0}\right)\left\langle N\left(k_{N}\right)\left|\left(J_{\sigma}{ }^{V}+J_{\sigma}^{A}\right)^{\star}\right| \beta\left(k_{N}+q\right)\right\rangle\left\langle\beta\left(k_{N}+q\right)\left|J_{\lambda}^{V}+J_{\lambda}^{A}\right| N\left(k_{N}\right)\right\rangle \\
& =\frac{M_{N}}{k_{N 0}}\left[\bar{A} \delta_{\lambda \sigma}+\bar{B} k_{N \lambda} k_{N \sigma}+\bar{C} \epsilon_{\lambda \sigma \gamma \delta} q_{\gamma} k_{N \delta}+\bar{D} q_{\lambda} q_{\sigma}+\bar{E}\left(q_{\lambda} k_{N \sigma}+q_{\sigma} k_{N \lambda}\right)\right],
\end{aligned}
$$

with $\bar{A}, \cdots, \bar{E}$ functions of $q^{2}$ and $W$. Time reversal and parity invariance rule out the presence of a term proportional to $q_{\lambda} k_{N \sigma}-q_{\sigma} k_{N \lambda}$ in Eq. (A1). Comparing Eq. (A1) with Eq. (41), in the laboratory frame, shows that

$$
\begin{gathered}
\bar{A}=\alpha\left(q^{2}, W\right), \quad M_{N}{ }^{2} \bar{B}=\beta\left(q^{2}, W\right), \quad M_{N} \bar{C}=\gamma\left(q^{2}, W\right), \quad \bar{D} \equiv \delta\left(q^{2}, W\right)=V_{2}\left(q^{2}, W\right)+A_{2}\left(q^{2}, W\right), \\
M_{N} \bar{E} \equiv \epsilon\left(q^{2}, W\right)=q_{0}^{-1}\left\{V_{1}\left(q^{2}, W\right)+A_{1}\left(q^{2}, W\right)+q^{2}\left[V_{2}\left(q^{2}, W\right)+A_{2}\left(q^{2}, W\right)\right]+\frac{1}{2}\left[I_{V}\left(q^{2}, W\right)+I_{A}\left(q^{2}, W\right)\right]\right\} .
\end{gathered}
$$

It is straightforward to calculate the contraction of $T_{\lambda \sigma}$ with the leptonic trace. We find that Eq. (13) and Eq. (22) for the strangeness-conserving case are replaced by

$$
\begin{aligned}
& d^{2} \sigma\left(\left(\begin{array}{l}
\nu \\
\bar{\nu}
\end{array}\right)+p \rightarrow\left(\begin{array}{l}
l \\
l
\end{array}\right)+\beta(S=0)\right) / d \Omega_{l} d E_{l}=\frac{G^{2} \cos ^{2} \theta_{C}}{(2 \pi)^{2}} \frac{\left[\left(E_{\nu}-q_{0}\right)^{2}-m_{l}^{2}\right]^{1 / 2}}{E_{\nu}} \kappa^{( \pm)}, \\
& d^{2} \sigma\left(\left(\begin{array}{l}
\nu \\
\bar{\nu}
\end{array}\right)+p \rightarrow\left(\begin{array}{l}
l \\
\bar{l}
\end{array}\right)+\beta(S=0)\right) / d\left(q^{2}\right) d q_{0}=\frac{G^{2} \cos ^{2} \theta_{C}}{4 \pi E_{\nu}{ }^{2}} \kappa^{( \pm)},
\end{aligned}
$$


with

$\kappa^{( \pm)}=\left(q^{2}+m_{l}^{2}\right) \alpha^{( \pm)}\left(q^{2}, W\right)+\left[2 E_{\nu}{ }^{2}-2 E_{\nu} q_{0}-\frac{1}{2}\left(q^{2}+m_{l}{ }^{2}\right)\right] \beta^{( \pm)}\left(q^{2}, W\right)$

$$
\mp\left[\left(2 E_{\nu}-q_{0}\right) q^{2}-m_{l}^{2} q_{0}\right] \gamma^{( \pm)}\left(q^{2}, W\right)+\frac{1}{2} m_{l}^{2}\left(q^{2}+m_{l}^{2}\right) \delta^{( \pm)}\left(q^{2}, W\right)-2 m_{l}^{2} E_{\nu} \epsilon^{( \pm)}\left(q^{2}, W\right) .
$$

Inspection of Eq. (A5) and its analog for a neutron target shows that $\beta^{( \pm)}, \gamma^{( \pm)}, \epsilon^{( \pm)}$, and $\alpha^{( \pm)}+\frac{1}{2} m_{l}{ }^{2} \delta^{( \pm)}$are independently measurable. Since the derivation of the sum rule on $\alpha^{( \pm)}$given in Sec. 4 shows that

$$
0=\int d q_{0}\left(\delta^{(-)}-\delta^{(+)}\right)
$$

we may modify Eq. (15) to read

$$
\begin{aligned}
C_{I}{ }^{1}+C_{I}{ }^{2}=(1 & \left.+q^{2} / 4 M_{N}^{2}\right) g_{A}\left(q^{2}\right)^{2}+\left(q^{2} / 4 M_{N}^{2}\right) g_{V}\left(q^{2}\right)^{2} \\
& +\frac{1}{2} m^{2}\left[\left(1+q^{2} / 4 M_{N}^{2}\right) f_{V}\left(q^{2}\right)^{2}-g_{V}\left(q^{2}\right) f_{V}\left(q^{2}\right) / M_{N}+\left(q^{2} / 4 M_{N}{ }^{2}\right) h_{A}\left(q^{2}\right)^{2}-h_{A}\left(q^{2}\right) g_{A}\left(q^{2}\right) / M_{N}\right] \\
& \quad+\int_{M_{N}+M_{\pi}}^{\infty} \frac{W}{M_{N}} d W\left[\alpha^{(-)}\left(q^{2}, W\right)+\frac{1}{2} m_{l}^{2} \delta^{(-)}\left(q^{2}, W\right)-\alpha^{(+)}\left(q^{2}, W\right)-\frac{1}{2} m_{l}^{2} \delta^{(+)}\left(q^{2}, W\right)\right] .
\end{aligned}
$$

Thus, in the strangeness-conserving case, when lepton-mass terms are included there are still three sum rules which may be directly compared with experiment.

In the strangeness-changing case, equations similar to Eqs. (A3)-(A5) hold, and $\beta_{(p, n)}{ }^{( \pm)}, m_{l}{ }^{2} \epsilon_{(p, n)}{ }^{( \pm)} \pm q^{2} \gamma_{(p, n)}{ }^{( \pm)}$, and $\alpha_{(p, n)}{ }^{( \pm)}+\frac{1}{2} m_{l}{ }^{2} \delta_{(p, n)}{ }^{( \pm)} \pm q_{0} \gamma_{(p, n)}{ }^{( \pm)}$are independently measurable. We see that in this case, when lepton-mass terms are included, only the sum rules on $\beta_{(p, n)}( \pm)$ can be directly compared with experiment.

It is easy to verify that the results of Eq. (26) and Eq. (27), referring to the high neutrino-energy behavior of neutrino cross sections, are unchanged by adding the lepton-mass terms. Equation (24) becomes

$$
\frac{d \sigma}{d q^{2}}=\int_{\left(q^{2} / 2 M_{N}\right)-}^{E_{\nu}\left(1-L / 4 E_{\nu}{ }^{2}\right)} d q_{0} \frac{d^{2} \sigma}{d\left(q^{2}\right) d q_{0}}, \quad L=q^{2}+m_{l}{ }^{2}+\frac{4 E_{\nu}{ }^{2} m_{l}{ }^{2}}{q^{2}+m_{l}{ }^{2}} .
$$

If, in addition to Eq. (25), we postulate that

$$
\int^{\infty} \frac{d q_{0}}{q_{0}^{2}}\left(\delta^{(-)}-\delta^{(+)}\right), \quad \int^{\infty} \frac{d q_{0}}{q_{0}}\left(\epsilon^{(-)}-\epsilon^{(+)}\right)
$$

are convergent (and similarly in the strangeness-changing case), then we immediately obtain Eqs. (26) and (27). 